\title{
Oral vs. intravenous empirical antimicrobial therapy in febrile neutropenic patients receiving childhood cancer chemotherapy
}

\author{
Ângela Rech Cagol, ${ }^{1}$ Cláudio Galvão de Castro Junior, ${ }^{2}$ Maria Cristina Martins, ${ }^{3}$ \\ Adão Leal Machado, ${ }^{4}$ Renato Chagas Ribeiro, ${ }^{5}$ Lauro J. Gregianin, ${ }^{6}$ Algemir Lunardi Brunetto ${ }^{7}$
}

\begin{abstract}
Objective: To compare the use of intravenous vs. oral antibiotic therapy.

Methods: All febrile neutropenic patients younger than 18 years old with low risk of complications and receiving chemotherapy were selected. The study was conducted from 2002 to 2005 at the Pediatric Oncology Unit of Hospital de Clínicas de Porto Alegre, Porto Alegre, Brazil. Patients were divided into group A and group B and were randomly assigned to receive oral or intravenous therapy. The empirical antimicrobial treatment used for group A consisted in oral ciprofloxacin plus amoxicillin-clavulanate and intravenous placebo, and group B received cefepime and oral placebo.

Results: A total of 91 consecutive episodes of febrile neutropenia in 58 children were included in the study. For patients of group A, treatment failure rate was $51.2 \%$; the mean length of hospital stay was 8 days (range $2-10$ days). For patients treated with intravenous antibiotic therapy, treatment failure rate was $45.8 \%$; the mean length of hospital stay was 7 days (range 3-10 days).

Conclusion: There was no difference in the outcome in oral vs. intravenous therapy. There is need of larger randomized trials before oral empirical therapy administered to this population should be considered the new standard of treatment.
\end{abstract}

J Pediatr (Rio J). 2009;85(6):531-535: Pediatrics, neutropenia, cancer.

\section{Introduction}

Until the past decade, almost all children with cancer who had an episode of febrile neutropenia were hospitalized and received intravenous broad-spectrum antibiotic regardless of their clinical condition. More recently, many researchers have demonstrated that febrile neutropenic patients comprise a heterogeneous group and may have several different levels of risk for complications related to the oncologic treatment. ${ }^{1}$ In the last few years, several studies have tried to identify clinical and laboratory factors that make it possible to detect, in a heterogeneous group of neutropenic patients,

1. Doutora, Oncologia Pediátrica, Universidade Federal do Rio Grande do Sul (UFRGS), Porto Alegre, RS, Brazil. Professora adjunta, Pediatria, Universidade de Caxias do Sul (UCS), Caxias do Sul, RS, Brazil. Médica oncologista pediátrica,Unidade de Oncologia Pediátrica, Hospital de Clínicas de Porto Alegre (HCPA), UFRGS, Porto Alegre, RS, Brazil.

2. Mestre, Oncologia Pediátrica, UFRGS, Porto Alegre, RS, Brazil. Médico oncologista pediátrico, Unidade de Oncologia Pediátrica, HCPA, UFRGS, Porto Alegre, RS, Brazil.

3. Médica oncologista pediátrica, Unidade de Oncologia Pediátrica, HCPA, UFRGS, Porto Alegre, RS, Brazil.

4. Mestre. Infectologista pediátrico, Unidade de Oncologia Pediátrica, HCPA, UFRGS, Porto Alegre, RS, Brazil.

5. Farmacêutico, Unidade de Oncologia Pediátrica, HCPA, UFRGS, Porto Alegre, RS, Brazil.

6. Doutor, Oncologia Pediátrica, UFRGS, Porto Alegre, RS. Médico oncologista pediátrico, Unidade de Oncologia Pediátrica, HCPA, UFRGS, Porto Alegre, RS, Brazil.

7. Doutor. Chefe, Serviço de Oncologia Pediátrica, HCPA, UFRGS, Porto Alegre, RS, Brazil.

No conflicts of interest declared concerning the publication of this article.

Suggested citation: Cagol AR, de Castro Junior CG, Martins MC, Machado AL, Ribeiro RC, Gregianin LJ, et al. Oral vs. intravenous empirical antimicrobial therapy in febrile neutropenic patients receiving childhood cancer chemotherapy. J Pediatr (Rio J). 2009;85(6):531-535.

Manuscript submitted Jun 8 2009, accepted for publication Sep 92009.

doi:10.2223/JPED.1956 
those at a higher risk of complications and those with good prognosis who usually present with quick fever reduction without severe complications. The identification of these factors may help to personalize treatment according to the risk factors of the febrile episode.

There is increasing evidence supporting the idea that neutropenic patients at low risk do not necessarily need long-term hospitalization and broad-spectrum antibiotic therapy for invasive bacterial infections. The strategies for this group include short-term antimicrobial treatments with consequent reduction of the length of hospital stay and outpatient management by means of oral antibiotics. ${ }^{2}$ Much of the evidence, however, is a result of studies with adults. Kern et al. ${ }^{3}$ and Freifeld et al. ${ }^{4}$ demonstrated the effectiveness and safety of oral antimicrobial therapy when compared with the parenteral regimen in clinical trials involving low-risk patients.

In addition to being as effective as intravenous therapy, oral therapy has shown advantages in comparison with intravenous therapy: 1) it does not require use of intravenous access; 2 ) it can be administered at outpatient clinics, which reduces the contact of patients with nosocomial pathogens; 3 ) it can improve quality of life; and 4) it might provide a significant reduction of costs. ${ }^{3}$

Studies showing that children with febrile neutropenia can be safely treated as outpatients are always welcome in pediatrics. We decided to use ciprofloxacin and amoxicillinclavulanate as the oral regimen because both agents are well absorbed when orally administered and because this association offers good coverage against gram-negative bacilli and gram-positive cocci. ${ }^{5}$ In a group of inpatients, we compared the effectiveness of the oral therapy vs. cefepime, which may be an alternative to intravenous monotherapy in patients with febrile neutropenia.

\section{Patients and methods Selection of patients}

Patients younger than 18 years old and with febrile neutropenia at low risk for invasive bacterial infection, treated with chemotherapy from September 2002 to April 2005 at the Pediatric Oncology Unit of Hospital de Clínicas de Porto Alegre, Porto Alegre, Brazil, were eligible for the present study. This was a prospective, randomized, double-blind, placebo-controlled study. For the analysis, we included at most three episodes of febrile neutropenia per patient. All children included in the study were receiving chemotherapy and none of them had indication for transplantation of hematopoietic stem cells. All patients received a central venous catheter that was implanted upon hospital admission. All patients signed a written consent form. This study was approved by the Research Ethics Committee of Hospital de Clínicas de Porto Alegre. If the patient was allocated to the study more than once, a signed written consent form was provided again.

\section{Definitions}

Neutropenia was defined as a neutrophil count of less than $1,000 / \mathrm{mcL}$, and fever was defined as a single axillary temperature measurement greater than or equal to $38.5^{\circ} \mathrm{C}$, or three measurements between 37 and $38.4{ }^{\circ} \mathrm{C}$ within a 24-hour period.

The authors defined patients as at low risk of infectious complications if they had febrile neutropenia, but did not have other comorbidities indicative of severity, such as bacterial septicemia, suspected central venous access infection, septic shock, metabolic instability, altered level of consciousness, bleeding requiring blood transfusion, dehydration, perirectal or soft tissue abscess, vomiting or organ failure. Chemotherapy dose intensity was not taken into account when classifying patients as low risk. Patients with solid tumors, including those with refractory or relapsed conditions, and patients with acute leukemia in the maintenance phase of treatment, were accepted for inclusion. Patients who had received hematopoietic stem cell transplantation and those with acute leukemia in the chemotherapy remission induction or consolidations phases were considered high risk and as such were not included in this study.

\section{Treatment and initial assessment}

The initial assessment covered clinical history, physical examinations and laboratory tests, intended to identify possible foci of infection. The laboratory tests included complete blood count, serum urea, creatinine, bilirubins and transaminases, blood culture via central venous access or via peripheral access, standard urine test, urine culture and chest X-ray.

Patients were admitted to the unit and then randomized to receive oral or intravenous treatment. Each patient could be enrolled on the study a maximum of three times - for discrete episodes of febrile neutropenia - randomized as new for each enrollment.

Randomization consisted of distributing patients into blocks of 10 , with selection made by a pharmacist who drew lots before patients were recruited. Patients were allocated to either group $A$ or group $B$, where patients in group $A$ were given oral antimicrobial treatment and those in group $B$ were given intravenous treatment. The lead researcher was blinded to the randomization results.

Group A was treated with $30 \mathrm{mg} / \mathrm{kg} /$ day of ciprofloxacin given orally every 12 hours (up to a maximum daily dose of $1,500 \mathrm{mg}$ ) in combination with $30 \mathrm{mg} / \mathrm{kg} /$ day of amoxycillin/ clavulanate, every 8 hours (up to a maximum daily dose of $1,500 \mathrm{mg}$ ), also given orally, plus an intravenous placebo. Group B was given 150 mg/kg/day of cefepime intravenously 
every 8 hours (up to a maximum daily dose of $4 \mathrm{~g}$ ), plus oral placebo.

\section{Clinical progress}

In both groups, patients were assessed daily. The duration of treatment and hospital discharge were decided once the fever had been controlled and the neutrophil count had recovered (i.e., the neutrophil count was greater than $1,000 / \mathrm{mcL}$ ), a negative blood culture had been taken and the patient had been free from fever for at least 48 hours. Monitoring included daily physical examinations aimed at identifying possible foci of infection and laboratory assessments including a complete blood count repeated every 3 days.

\section{Outcomes}

Cases were considered unsuccessful if one or more of the following conditions indicative of invasive bacterial infection was observed: 1) hemodynamic instability unrelated to lost volume; 2) fever that had not abated 72 hours after starting antibiotics; 3 ) repeat episode of fever lasting at least 24 hours and occurring after the 48-hour period with no fever; 4) death during infection; 5) grade III and IV vomiting; and 6) infections that demanded the addition of antibiotics not included in the study protocol.

Treatment was considered successful if none of the above following conditions indicative of invasive bacterial infection was observed and the patient completed the treatment without any need to adjust their antimicrobial treatment.

If a specific pathogen was identified, the study was opened, and treatment was adjusted in accordance with blood culture and antibiogram, except for in cases when the patient was clinically stable and free from fever, in which case the treatment defined by the study protocol was continued.

\section{Statistical analysis}

The sample size was calculated on the basis of an expected failure rate of $20 \%$ for the intravenous treatment vs. $50 \%$ for patients treated with orally administered drugs. Setting the type I error (alpha) at 5\% and the statistical power at $80 \%$, a sample size of 45 episodes per group was arrived at.

Continuous variables were presented as medians, means and interquartile ranges (IQ 25-75\%). Comparisons were made using the Mann-Whitney test. Categorical variables were presented as figures and percentages and were compared using the chi-square test or Fisher's exact test, as necessary. The level of statistical significance was set at alpha $=0.05$. Data were analyzed using SPSS version 14.0 .

\section{Results}

There were a total of 91 episodes of febrile neutropenia distributed across the 58 children enrolled on the study. The patients' characteristics are given in Table 1. All patients had neutrophil counts below $1,000 / \mathrm{mcL}$.

The patients enrolled on the study had the following diagnoses: osteosarcoma (10 patients), primitive neuroectodermal tumor of the central nervous system (seven), Wilms' tumor (seven), rhabdomyosarcoma (six), soft tissue sarcoma (seven), leukemia (seven), hepatoblastoma (two), neuroblastoma (six), Ewing sarcoma (two), retinoblastoma (two), lymphoma (one) and gonadal tumor (one).

In group $A$, the rate of ineffectiveness was $51.2 \%$. The mean length of hospital stay, for all patients in this group, was 8 days (variation of $2-10$ days). The events that led to changing the treatment regimen were: grade III/IV vomiting (three cases), fever lasting for more than 72 hours (seven episodes), positive blood culture (four episodes), mucositis severe (one episode), anal cellulitis (one episode) and no obvious cause (three episodes).

The rate of treatment failure in group B was $45.8 \%$, which is greater than the estimated rate used for the initial calculation. The authors had not expected to observe so many failures in this group and the elevated number is probably due to their not having taken chemotherapy dosage intensity or disease stage into account when allocating patients. The mean length of hospital stay in this group was 7 days (variation of 3-10 days). Events that caused the antibiotic to be changed were: grade III and IV vomiting (one episode), fever lasting for more than 72 hours ( 15 episodes), positive blood culture (four episodes) and diarrhea (one episode).

When the two groups' treatment failure rates were compared, it was observed that the gap between the oral treatment group (51.2\%) and the intravenous treatment group $(45.8 \%)$ was very narrow and was not statistically significant (difference: $5.4 ; 95 \%$ confidence interval: -17.3 to $28.1 ; p=0.77$ ).

\section{Discussion}

The best strategy for treating febrile neutropenia in patients with cancer has not yet been entirely understood, and studies examining whether oral administration is effective are welcome.

A number of studies undertaken with adult patients have found that oral administration is an acceptable alternative for low-risk febrile neutropenia patients. Klastersky et al. ${ }^{6}$ demonstrated that oral administration of ciprofloxacin and amoxycillin/clavulanate allows to discharge patients who are at low risk of infectious complications from hospital earlier. 
Table 1 - Characteristics and outcomes broken down by treatment regimen

\begin{tabular}{|c|c|c|c|}
\hline \multirow[b]{2}{*}{ Characteristics } & \multicolumn{2}{|c|}{ Antibiotic administration route } & \multirow[b]{2}{*}{$\mathbf{p}$} \\
\hline & Oral, $n=43$ & Intravenous, $n=48$ & \\
\hline Age (years) & $7.9 \pm 4.3$ & $7.6 \pm 4.8$ & \\
\hline Mean (minimum-maximum) & $6.0(4.4-11.4)$ & $6.2(3.4-11.7)$ & $0.50 *$ \\
\hline Male sex, n (\%) & $26(60.5)$ & $31(64.6)$ & $0.85^{+}$ \\
\hline \multicolumn{4}{|l|}{ Disease, n (\%) } \\
\hline Solid tumor & $39(90.7)$ & $43(89.6)$ & \\
\hline Neoplasm hematological & $4(9.3)$ & $5(10.4)$ & $0.99^{+}$ \\
\hline Neutrophil count / mcL < 1,000, n (\%) & $43(100.0)$ & $48(100.0)$ & $0.99^{+}$ \\
\hline \multicolumn{4}{|l|}{ Reasons for switching regimen, $\mathrm{n}(\%)$} \\
\hline Persistent fever & $7(16.3)$ & $15(31.3)$ & $0.16^{+}$ \\
\hline Positive culture & $4(9.3)$ & $4(8.3)$ & $0.99^{\ddagger}$ \\
\hline Vomiting & $3(7.0)$ & $1(2.1)$ & $0.34^{\neq}$ \\
\hline Mucositis severe & $1(2.3)$ & - & $0.47^{\ddagger}$ \\
\hline Anal cellulitis & $1(2.3)$ & - & $0.47^{\ddagger}$ \\
\hline Diarrhea & - & $1(2.1)$ & $0.99^{\neq}$ \\
\hline No obvious reasons & $3(7.0)$ & - & $0.10^{\neq}$ \\
\hline Mean length of hospital stay in days (variation) & $8.0(2.0-10.0)$ & $7.0(3.0-10.0)$ & 0.37 \\
\hline Treatment failure, n (\%) & $22(51.2)$ & $22(45.8)$ & $0.77^{+}$ \\
\hline
\end{tabular}

The safety of orally administered antibiotics has also been demonstrated with children suffering from febrile neutropenia. Paganini et al. ${ }^{7}$ treated 175 episodes of low risk febrile neutropenia and showed that ciprofloxacin given orally to outpatients 24 hours after a single dose of an intravenous ceftriaxone and amikacin regimen was as safe and effective as parenteral ceftriaxone use. Gupta et al. ${ }^{8}$ published a randomized study comparing the efficacy of oral administration of amoxycillin/clavulanate plus ofloxacin with intravenous administration of ceftriaxone and amikacin (infused once daily) in low risk pediatric outpatients with febrile neutropenia and observed that both outpatients regimens were safe and effective, with similar success rates in the oral and intravenous subsets. A Brazilian study that recruited low-risk children with neutropenia and solid tumors or stage I or II lymphoma demonstrated that these cases had a favorable clinical course, whether given oral ciprofloxacin or intravenous ceftriaxone, and there were no deaths from infection during the study. ${ }^{9}$ In our study, success rates and outcomes were similar for both regimens.

The objective of this study was to assess the efficacy of oral vs. intravenous antibiotics for patients with febrile neutropenia. The results did not detect a statistically significant difference between the two groups. However, the confidence interval extended from -17.3 to 28.1 . Therefore, a greater number of episodes of febrile neutropenia could have made a stronger contribution to supporting the initial hypothesis of this research.
Criteria for identifying children at low risk of infection are not yet fully defined, but both clinical and laboratory risk factors can be useful. Currently, the following are taken into account when defining risk groups at: diagnosis, cancer status, chemotherapy dose-intensity, comorbidities and duration of neutropenia. The Multinational Association of Supportive Care in Cancer (MASCC) score $^{1}$ has often been used, but it was validated with adult patients only. There have been few studies of children with febrile neutropenia defining which patients are low and which are high risk. ${ }^{10}$ More recently, procalcitonin and C-reactive protein concentrations in plasma have been suggested as a marker for estimating severity of infection and as a potential aid for choosing initial treatment. ${ }^{11}$ We did not assay this marker in our study.

For this study, we excluded patients with acute leukemia (with the exception of those already in the maintenance phase) and patients with stage III or IV Iymphoma. In contrast with previous studies, we did not include the status of solid tumors or the intensity of chemotherapy dosage in our exclusion criteria for patients selected as low risk. All patients with solid tumors, including those with refractory or relapsed disease, were selected for the study.

Earlier studies with febrile neutropenia patients have listed the following pathogens as being the most commonly identified within this patient population: Escherichia coli, Klebsiella pneumoniae, Staphylococcus aureus and coagulase-negative Staphylococcus. ${ }^{12}$ Ciprofloxacin and other fluoroquinolones can be used to manage these patients, 
demonstrating excellent efficacy against the most often identified gram-negative bacteria. ${ }^{12}$ One of the limitations of fluoroquinolones is inadequate coverage of many grampositive organisms. ${ }^{12}$ Therefore, for this study, we decided to combine amoxycillin with clavulanate, based on its excellent tolerability and its relatively wide spectrum.

One factor limiting oral treatment is the presence of mucositis or enteritis, which is provoked by chemotherapy in a significant fraction of these patients and which can reduce their tolerance of oral antibiotics. ${ }^{13}$ In our study, just one patient suffered diarrhea, one mucositis and one had anal cellulitis, all in group A, while in group $B$ this type of toxicity did not occur.

Vomiting is also considered a limitation to using oral antibiotics for febrile neutropenia patients. In our study, there were three episodes of grade III vomiting in group $A$ and one episode of grade III vomiting in group $B$, causing a change from oral to intravenous treatment (bear in mind that the author was unaware which group each patient was in when she detected this toxicity). It was, nevertheless, considered that there was good tolerance of the oral regimen in our study.

Criteria for the management of febrile neutropenia patients can be found in the literature, relating to when we should rethink the antimicrobial treatment being given to patients with persistent fever. If fever lasts more than three to five days after starting treatment, in patients whose infection or foci have not yet been identified, suspicion should be aroused that the infection is not bacterial, that it is resistant to the antimicrobial regimen being used or, even, that it responds slowly to treatment, or that serum antibiotic concentrations are inadequate. At this point a wide-ranging review of cultures, a meticulous physical examination, chest $\mathrm{X}$-rays, investigation of the central catheter, imaging studies of any organ suspected of infection and blood samples or specimens from suspected sites of infection are all indicated. In our study, after 72 hours of persistent fever, or if fever returned after a period free from fever, it was recommended that the antibiotic be changed and the study regimen was considered to have failed.

Because outpatient management of pediatric patients with cancer and febrile neutropenia is associated with reduced costs and increased quality of life, it is a valid objective for studies to attempt to confirm ever further the efficacy of oral regimens for low-risk patients. However, the objective of this study was not to assess management of treatment outside of the hospital setting, quality of life or costs, but to evaluate the efficacy of oral treatment versus intravenous treatment. Although this study has shown that empirical oral treatment pays a similar role to intravenous treatment for low risk febrile neutropenia patients, randomized studies with larger patient samples are needed before the oral treatment can be made standard for this group of patients.

\section{Acknowledgements}

We would like to thank the Instituto de Câncer Infantil do Rio Grande do Sul for their financial support and the pediatric oncology team at the Hospital de Clínicas for their help with conducting this study.

\section{References}

1. Klastersky J, Paesmans M, Rubenstein EB, et al. The Multinational Association for Supportive Care in Cancer risk index: A multinational scoring system for identifying low-risk febrile neutropenic cancer patients. J Clin Oncol. 2000;18:3038-51.

2. Santolaya ME, Alvarez AM, Avilés EL, et al. Early hospital discharge followed by outpatient management versus continued hospitalization of children with cancer, fever, and neutropenic at low risk for invasive bacterial infection. J Clin Oncol. 2004; 22:3784-9.

3. Kern WV, Cometta A, de Bock R, et al. Oral versus intravenous empiric therapy for fever in patients with granulocytopenia who are receiving cancer chemotherapy. N Engl J Med. 1999; 341:312-8.

4. Freifeld A, Marchigiani D, Walsh T, et al. A double-blind comparison of empirical oral and intravenous antibiotic therapy for low-risk febrile patients with neutropenia during cancer chemotherapy. N Engl J Med. 1999; 341:305-11.

5. Shah A, Lettieri J, Kaiser L, et al. Comparative pharmacokinetics and safety of ciprofloxacin $400 \mathrm{mg}$ i.v. thrice daily versus $750 \mathrm{mg}$ po twice daily. J Antimicrob Chemother. 1994; 33:795-801.

6. Klastersky J, Paesmans M, Georgala A, et al. Outpatient oral antibiotics for febrile neutropenic cancer patients using a score predictive for complications. J Clin Oncol. 2006; 24: 4129-34.

7. Paganini H, Gómez S, Ruvinsky S, et al. Outpatient, sequential, parenteral-oral antibiotic therapy for lower risk febrile neutropenia in children with malignant disease. Cancer. 2003; 97:1775-80.

8. Gupta A, Swaroop C, Agarwala S, Bakhshi S. Randomized controlled trial comparing efficacy of oral amoxicillin-clavulanate and ofloxacin with intravenous ceftriaxone and amikacin as outpatient therapy in pediatric low risk febrile neutropenia. 2008 ASCO Annual Meeting. 2008; Abstract No 10020.

9. Petrilli AS, Dantas LS, Campos MC, et al. Oral Ciprofloxacin vs intravenous ceftriaxone administered in an outpatient setting for fever and neutropenia in low-risk pediatric oncology patients: randomized prospective trial. Med Ped Oncol. 2000; 34:87-91.

10. Wicki S, Keisker A, Aebi C, Leibundgut K, Hirt A, Ammann RA. Risk prediction of fever in neutropenia in children with cancer: a step towards individually tailored supportive therapy? Pediatr Blood Cancer. 2008;51:778-783.

11. Secmeer G, Devrim I, Kara A, et al. Role of procalcitonin and CRP in differentiating a stable from a deteriorating clinical course in pediatric febrile neutropenia. J Pediatr Hematol Oncol. 2007; 29:107-11.

12. Mullen CA. Ciprofloxacin in treatment of fever and neutropenia in pediatric cancer patients. Ped Infect Dis J. 2003; 22:1138-42.

13. Castagnola E, Boni L, Giachino M, et al. A multicenter, randomized, double-blind placebo-controlled trial of amoxicillin/clavulanate for the prophylaxis of fever and infection in neutropenic children with cancer. Pediatr Infect Dis J. 2003; 22:359-65.

Correspondence:

Algemir Lunardi Brunetto

Hospital de Clínicas de Porto Alegre,

Unidade de Oncologia Pediátrica

Ramiro Barcellos, 2350, 30 leste

CEP 90035-003 - Porto Alegre, RS - Brazi

Tel.: +55 (51) 3330.8087

Fax: +55 (51) 3330.8087

E-mail: abrunetto@hcpa.ufrgs.br 